\title{
Complete response associated with immune checkpoint inhibitors in advanced non-small-cell lung cancer: a meta-analysis of nine randomized controlled trials
}

This article was published in the following Dove Medical Press journal: Cancer Management and Research

Jie $\mathrm{Li}$

Qi He

Xiu Yu

Khalid Khan

Xuanwen Weng

Minjie Guan

Department of Respiratory Diseases, Second Clinical Medical College Shenzhen People's Hospital, Jinan University, Shenzhen 518020, China
Correspondence: Jie Li

Department of Respiratory Diseases, Second Clinical Medical College Shenzhen People's Hospital, Jinan University, 1017 Dongmen North Road, Luohu District, Shenzhen 518020, Guangdong, China

Tel +867552553 3018

$\mathrm{Fax}+8675525533497$

Email li_jie2018@।26.com
Purpose: The purposes of this study were to investigate whether the use of immune checkpoint inhibitors (ICIs) in advanced non-small-cell lung cancer (NSCLC) would increase the possibility of archiving complete response (CR) and assess the surrogate end points for overall survival (OS). Methods: We calculated the incidence and relative risk (RR) of CR events in patients assigned to ICIs compared to that in controls. Simple linear regression models were fitted for median OS and each surrogate (median progression-free survival [PFS], CRs, and objective response rate [ORR]).

Results: A total of 4,803 NSCLC patients from nine randomized controlled trials (RCTs) were included for analysis. The incidence of CR in NSCLC patients treated with ICIs was $1.5 \%(95 \%$ CI: $0.8-3.0)$ compared to $0.7 \%$ (95\% CI: 0.4-1.2) in chemotherapy (CT) groups. The use of ICIs in advanced NSCLC significantly improved the possibility of archiving CR (RR 2.89, 95\% CI: 1.44-5.81, $P=0.003$ ) compared to CT. Subgroup analysis according to ICIs showed that the use of atezolizumab (RR 3.26, $P=0.01$ ) and nivolumab (RR 4.83, $P=0.042$ ) in advanced NSCLC significantly improved the $\mathrm{CR}$ rate in comparison with $\mathrm{CT}$ alone, but not pembrolizumab and ipilimumab. We also found that the use of ICIs as first-line (RR 2.39, 95\% CI: $1.08-5.3, P=0.032$ ) or second-line (RR 4.99, 95\% CI: $1.10-22.66, P=0.038$ ) therapy significantly increased the change in obtaining a CR. In addition, correlation analysis indicates that PFS was strongly correlated with OS in NSCLC patients who received ICIs ( $r=0.89$ for PFS, $P=0.017$ ). No marked correlation was found between OS and CR $(r=0.19, P=0.75)$ and OS and ORR $(r=0.52, P=0.28)$. Conclusion: The CR is a rate event in advanced NSCLC, but the use of ICIs significantly increases the possibility of archiving CR in comparison with CT. PFS is significantly correlated with OS and could be used as a surrogate end point, but not for CRs and ORRs.

Keywords: immune checkpoint inhibitors, non-small-cell lung cancer, complete response, randomized controlled trials, meta-analysis, immunotherapy therapy, systematic review

\section{Introduction}

Lung cancer remains the leading cause of cancer deaths worldwide. ${ }^{1}$ In total, $80 \%-85 \%$ of lung cancer cases can be classified as non-small-cell lung cancer (NSCLC). ${ }^{2}$ Recent advances in the understanding of the pathogenesis of NSCLC have led to the introduction of a variety of biological agents into clinical practice. Small molecular inhibitors targeting NSCLC associated with driver mutations, such as EGFR or ALK inhibitors, have shown efficacy in controlling diseases. ${ }^{3-5}$ However, most of these patients would eventually develop drug resistance and a large number of NSCLC patients are presented 
without actionable mutations, and limited treatment options are available for this patient population. ${ }^{6-9}$ To address this issue, a novel treatment strategy is clearly needed.

The immune system has its inherent immunosuppressive mechanisms or uses checkpoints to limit prolonged immune activation and inflammation, which could be detrimental to the host. ${ }^{10}$ Tumors have co-opted these endogenous mechanisms as a means to evade immune surveillance by the host immune system. During the past decade, novel drugs targeting checkpoint pathways leading to a reinvigorated antitumor immune response have shown promising efficacy in advanced NSCLC. ${ }^{10,11}$ Among immune therapies, the programmed cell death 1 (PD-1) and the programmed cell death receptor 1 (PD-L1) blockade therapies are the most investigated. Preclinical studies have demonstrated that PD-1 receptor has emerged as a dominant negative regulator of antitumor T-cell effector function when engaged by its ligand PD-L1. ${ }^{12,13}$ Further works found that inflammation-induced PD-L1 expression in the tumor micro-environment results in PD-1-mediated T-cell exhaustion, inhibiting the antitumor cytotoxic T-cell response. As a result, PD-1 pathway blockade by using anti-PD-1 or antiPD-L1 drugs could relieve immune suppression of antitumor T cells, which results in tumor cell death. ${ }^{14,15}$ Currently, three immune checkpoint inhibitors (ICIs), namely, nivolumab (anti-PD-1), pembrolizumab (anti-PD-L1), and atezolizumab (anti-PD-L1), have been approved for either first-line or subsequent therapy in advanced NSCLC. ${ }^{16-19}$ Although these agents have shown greater activity, in terms of progression-free survival (PFS) or overall survival (OS), compared to controlled therapies, specifically when compared to placebo, a clinically relevant increase in complete response (CR) is not reported and the role of ICIs in increasing the curability of this cancer remains unclear. We thus conducted this meta-analysis of published reports about ICI-containing regimens vs chemotherapy (CT) alone to investigate the incidence rates and relative risk (RR) of CR in advanced NSCLC patients.

\section{Methods}

\section{Selection of studies}

To identify studies for inclusion in our systematic review and meta-analysis, we did a broad search of four databases, including Embase, PubMed/MEDLINE, the Cochrane Central Register of Controlled Trials, and the Cochrane Database of Systematic Reviews, from the date of inception of every database to August 2018. The search was limited to human studies and randomized controlled trials (RCTs). No language restriction was imposed. If more than one publication was found for the same trial, the most recent was considered for analysis. Abstracts of the American Society of Clinical Oncology (ASCO), the European Society for Medical Oncology Congress (ESMO) since 2002, and the World Gastrointestinal Congress since 2006 were also searched manually.

To be eligible for inclusion in our systematic review and meta-analysis, study populations (referred to hereafter as cohorts) had to meet all the following criteria: 1) patients with pathologically confirmed NSCLC; 2) prospective RCTs assessing patients received systematic therapy with or without ICIs; and 3) reported outcomes of interest (ie, tumor control and survival). We did not restrict our search to language and country. We excluded case reports with fewer than five patients, reviews, notes, letters, errata, and commentaries.

\section{Definition of outcomes}

Treatment with ICIs was considered as the experimental arms and the other treatments as the standard comparators. CRs were considered as the main outcomes, and the analysis was conducted in order to find a significant difference between the two arms. CRs were defined as disappearance of all target lesions. Any pathological lymph nodes (whether targeted or non-targeted) must have reduction in the short axis to $<10$ $\mathrm{mm}$ according to response evaluation criteria in solid tumors (RECIST) criteria. OS was defined as the period from starting targeted therapy until death or last follow-up. Time to progression (TTP)/PFS was defined as the period from starting targeted therapy until progression or last follow-up, and objective response rate (ORR) was defined as rate of partial responses and CRs.

\section{Data extraction}

Two authors conducted the data extraction independently. It was performed according to the PRISMA statement, ${ }^{20}$ and any types of discrepancies were resolved by consensus. The data extracted for each trial were first author's name, year of publication, number of enrolled patients, dose of ICIs, median age, median OS, and median PFS.

\section{Statistical method}

For calculating the incidence, the number of patients with $\mathrm{CR}$ and the number of patients treated in each arm were extracted from the efficacy profile of the selected trials. The proportion of patients with CR and the derived 95\% CI were calculated for each study. We also calculated the RR and the CIs of events in patients assigned to ICIs compared to the controlled patients in the same study. To calculate the 95\% CIs, the variance of a log-transformed study-specific RR was derived using the delta method. ${ }^{21}$ Between-study 
heterogeneity was estimated using the $\chi^{2}$-based Q-statistic. ${ }^{22}$ Heterogeneity was considered as statistically significant when $P_{\text {heterogeneity }}$ was $<0.1$. When substantial heterogeneity was observed, the pooled estimate, calculated based on the random-effects model, was reported using the method described by Dersimonian and Laird, ${ }^{23}$ which considered both within- and between-study variations. We also conducted the prespecified subgroup analyses according to treatment line and specific ICIs. We assessed potential publication bias by visual inspection of the symmetry of funnel plots and with tests described by Begg and Mazumdar ${ }^{24}$ and Egger et al. ${ }^{25}$ Study quality was assessed by using the Jadad five-item scale that included the randomization, double blinding, and withdrawals; the final score was reported between 0 and $5 .^{26}$ All data were collected using Microsoft Office Excel 2003; and meta-analysis was performed using open Meta-Analyst software version 4.16 .12 (http://www.cebm.brown.edu/ openmeta, 2018.09.13 last update).

\section{Ethics approval and consent to participate}

This meta-analysis was approved by the Ethical Committee of Shenzhen People's Hospital. The need for informed patient consent for inclusion was waived.

\section{Results}

A total of 350 studies were identified from the database search, of which 50 were duplicates and 285 did not meet the inclusion criteria and were therefore excluded. Thus, 15 reports were retrieved for full-text evaluation. A total of nine trials met the inclusion criteria and were included in this systematic review (Figure 1). The characteristics of patients and studies are listed in Table 1. Overall, a total of 4,803 NSCLC patients from nine RCTs were included for analysis. ${ }^{18,19,27-33}$ The median number of patients included in each study was 616 patients (range: $123-800$ patients). One trial was a placebo-controlled double blind trial, ${ }^{19}$ and the other eight trials were open-label randomized trials. ${ }^{18,27-33}$ Dosages for each molecule are reported in Table 1. Among the included trials, six trials investigated the role of ICIs as first-line therapy for advanced NSCLC, while the other two trials assessed the efficacy of ICIs in second-line settings. The quality of each included study was roughly assessed according to Jadad scale: one trial had a Jadad score of 5 and eight trials had a Jadad score of 3.

\section{Incidence of CR}

CRs were reported in 31 out of 2,067 patients in the experimental arm, with an incidence of $1.5 \%$ (95\% CI: $0.8-3.0)$, compared to 10 out of 1,836 patients treated in the control arm, with an incidence of $0.7 \%$ (95\% CI: 0.4-1.2).

\section{RR of CRs}

A total of 4,003 patients from seven RCTs were included for analysis. The RR of CR was 2.89 (95\% CI: 1.44-5.81, $P=0.003$; Figure 2) in patients treated with ICIs when

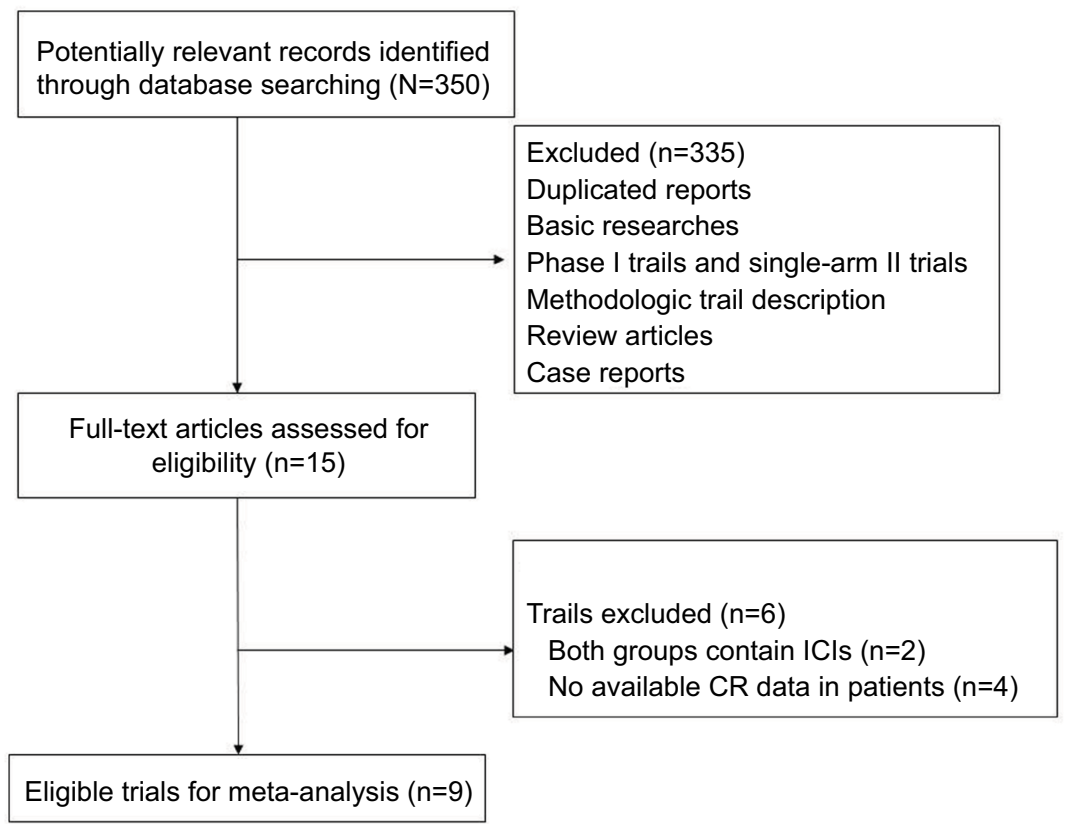

Figure I Selection process of RCTs included in the meta-analysis.

Abbreviations: CR, complete response; ICls, immune checkpoint inhibitors; RCT, randomized controlled trial. 
Table I Baseline characteristics of nine included trials

\begin{tabular}{|c|c|c|c|c|c|c|c|c|}
\hline Study/year & $\begin{array}{l}\text { Treatment } \\
\text { line }\end{array}$ & Total & $\begin{array}{l}\text { Treatment } \\
\text { arms }\end{array}$ & $\begin{array}{l}\text { Median age } \\
\text { (years) }\end{array}$ & $\begin{array}{l}\text { Median PFS } \\
\text { (months) }\end{array}$ & $\begin{array}{l}\text { Median OS } \\
\text { (months) }\end{array}$ & CR & $\begin{array}{l}\text { No. for } \\
\text { analysis }\end{array}$ \\
\hline \multirow[t]{2}{*}{ Socinski et $\mathrm{a}^{33} / 2018$} & First line & 800 & Atezolizumab+bevacizumab+CT & 63 & 8.3 & 19.2 & 13 & 353 \\
\hline & & & Bevacizumab $+\mathrm{CT}$ & 63 & 6.8 & 14.7 & 4 & 331 \\
\hline \multirow[t]{2}{*}{ Hellmann et $\mathrm{a}^{32} / 2018$} & First line & 299 & Nivolumab+ipilimumab & 64 & 7.2 & NR & 5 & 139 \\
\hline & & & $\mathrm{CT}$ & 64 & 5.5 & NR & 1 & 160 \\
\hline \multirow[t]{2}{*}{ Gandhi et al ${ }^{16} / 2018$} & First line & 616 & Pembrolizumab+CT & 65 & 9 & NR & 2 & 410 \\
\hline & & & $\mathrm{CT}$ & 63.5 & 6.7 & 11.3 & I & 206 \\
\hline \multirow{2}{*}{$\begin{array}{l}\text { Rittmeyer } \\
\text { et } \mathrm{a}^{29} / 2017\end{array}$} & Second line & 850 & Atezolizumab & 63 & 2.8 & 13.8 & 6 & 425 \\
\hline & & & Docetaxel & 64 & 4 & 9.6 & 1 & 425 \\
\hline \multirow[t]{2}{*}{ Govindan et al ${ }^{19} / 2017$} & First line & 956 & Ipilimumab+CT & 64 & 5.6 & 13.4 & 1 & 388 \\
\hline & & & Placebo $+C T$ & 64 & 5.6 & 12.4 & 2 & 361 \\
\hline \multirow[t]{2}{*}{ Reck et a $\left.\right|^{31} / 2016$} & First line & 305 & Pembrolizumab & 64.5 & 10.3 & NR & NR & 154 \\
\hline & & & $\mathrm{CT}$ & 66 & 6 & NR & NR & 151 \\
\hline \multirow[t]{2}{*}{ Langer et $\mathrm{al}^{31} / 2016$} & First line & 123 & Pembrolizumab+CT & 62.5 & 13 & NR & 0 & 60 \\
\hline & & & $\mathrm{CT}$ & 63.2 & 8.9 & NR & 0 & 63 \\
\hline \multirow[t]{2}{*}{ Brahmer et $\mathrm{al}^{27} / 2015$} & Second line & 272 & Nivolumab & 62 & 3.5 & 9.2 & NR & 135 \\
\hline & & & Docetaxel & 64 & 2.8 & 6 & $N R$ & 137 \\
\hline \multirow[t]{2}{*}{ Borghaei et al ${ }^{18} / 2015$} & Second line & 582 & Nivolumab & 61 & 2.3 & 12.2 & 4 & 292 \\
\hline & & & Docetaxel & 64 & 4.2 & 9.4 & 1 & 290 \\
\hline
\end{tabular}

Abbreviations: CR, complete response; CT, chemotherapy; NR, not reported; OS, overall survival; PFS, progression-free survival.

Studies
Socinski M.A. et al $/ 2018$
Rittmeyer A. et al/2017
Subgroup atezolizumab $\left(l^{2}=\mathrm{NA}, P=0.576\right)$
Hellmann M.D. et al/2018
Borghaei H.et al $/ 2015$
Subgroup nivolumab $\left(I^{2}=\mathrm{NA}, P=0.812\right)$
Gandhi L. et al/2018
Langer C.J. et al/2016
Subgroup pembrolizumab $\left(I^{2}=\mathrm{NA}, P=1.000\right)$
Govindan R. et al/2017
Subgroup iplimumab $\left(I^{2}=\mathrm{NA}, P=\mathrm{NA}\right)$
Overall $\left(l^{2}=\mathrm{NA}, P=0.560\right)$

Estimate $(95 \% \mathrm{Cl}) \quad$ Event/treatment Event/control

$\begin{array}{rrrr}3.047 & (1.004,9.252) & 13 / 353 & 4 / 331 \\ 6.000 & (0.725,49.625) & 6 / 425 & 1 / 425 \\ 3.623 & (1.365,9.619) & 19 / 778 & 5 / 756 \\ & & & \\ 5.755 & (0.681,48.672) & 5 / 139 & 1 / 160 \\ 3.973 & (0.447,35.329) & 4 / 292 & 1 / 290 \\ 4.830 & (1.055,22.114) & 9 / 431 & 2 / 450 \\ & & & \\ 1.005 & (0.092,11.017) & 2 / 410 & 1 / 206 \\ 1.049 & (0.021,52.051) & 0 / 60 & 0 / 63 \\ 1.005 & (0.092,11.017) & 2 / 470 & 1 / 269 \\ & & & \\ 0.465 & (0.042,5.108) & 1 / 388 & 2 / 361 \\ 0.465 & (0.042,5.108) & 1 / 388 & 2 / 361 \\ & & & \\ 2.888 & (1.436,5.807) & 31 / 2,067 & 10 / 1,836\end{array}$

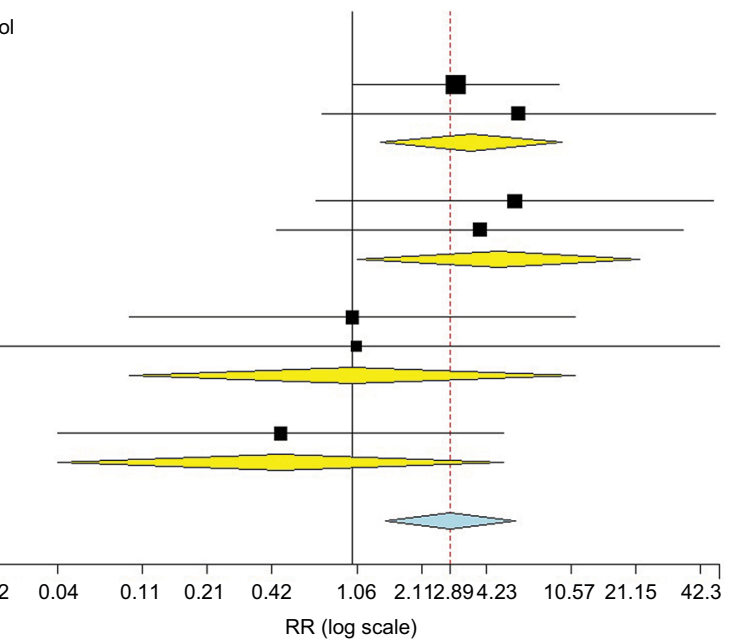

Figure 2 Subgroup analysis based on treatment line for RR of CR associated with ICls vs CT.

Abbreviations: $\mathrm{CR}$, complete response; $\mathrm{CT}$, chemotherapy; $\mathrm{ICI}$, immune checkpoint inhibitor; NA, not available.

compared to the controls, according to the fixed-effects model $\left(P=0.56, I^{2}=0 \%\right)$. We then performed subgroup analysis according to treatment line and showed that the use of ICIs as first-line (RR 2.39, 95\% CI: $1.08-5.3, P=0.032$ ) or second-line (RR 4.99, 95\% CI: 1.10-22.66, $P=0.038$ ) therapy significantly increased the chance of obtaining a $\mathrm{CR}$. Of note, the occasional wide variation in the CIs indicated that more trials were still needed to confirm our findings, although there was an improved RR of archiving CR in ICIs groups. In addition, subgroup analysis to specific ICIs showed that use of atezolizumab (RR 3.26, $P=0.01$ ) and nivolumab (RR 4.83, $P=0.042)$ in advanced NSCLC significantly improved the $\mathrm{CR}$ rate in comparison with $\mathrm{CT}$ alone, but not pembrolizumab and ipilimumab.

\section{Publication bias}

No evidence of publication bias was detected for the RR of CRs in this study by funnel plots, Begg's test $(P=0.40)$, and Egger's test $(P=0.57)$.

\section{Correlation between OS and PFS/ORR}

Data from nine cohorts were available for correlation analysis between OS and PFS. There was a strong correlation between median OS and median PFS ( $r=0.89)$, and this correlation 


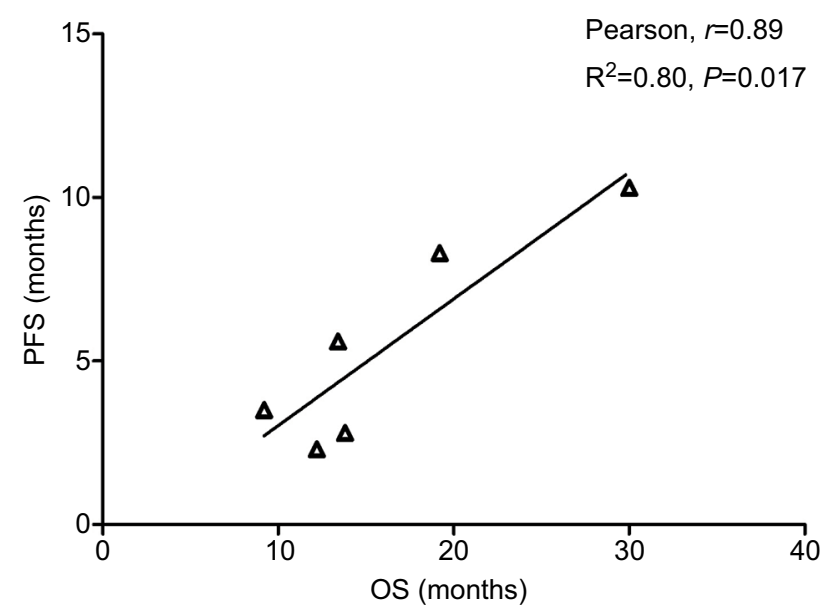

Figure 3 Correlation between median OS and PFS in NSCLC patients who received $\mathrm{ICls}$.

Abbreviations: $\mathrm{ICl}$, immune checkpoint inhibitor; NSCLC, non-small-cell lung cancer; OS, overall survival; PFS, progression-free survival.

was statistically significant ( $P=0.017$; Figure 3 ). Data from nine cohorts were available for correlation analysis between median OS and ORR/CRs. No marked correlation was found between median OS and CR ( $r=0.19, P=0.75$; Figure 4$)$ and median OS and ORR ( $r=0.52, P=0.28$; Figure 5$)$.

\section{Discussion}

During the past decades, major advance in cytotoxic agents has been achieved for advanced NSCLC. ${ }^{34}$ However, only a small number of advanced NSCLC patients receiving cytotoxic agents can achieve $\mathrm{CR}$. In recent years, immune therapy has significantly revolute treatment strategy for solid tumors including NSCLC. ${ }^{27,35}$ Although several case reports have been published, overall incidence and likelihood of achieving a CR in NSCLC receiving ICIs has not been systematically determined. In addition, obtaining a CR is independently associated with improved survival, not only for NSCLC but also for other solid and hematologic malignancies. As a result, it is of particular importance to determine whether the use of ICIs would increase the CR events in NSCLC patients.

To our best knowledge, this is the largest meta-analysis to specially investigate the possibility of archiving CRs associated with ICIs in the treatment of advanced NSCLC. A total of 4,803 NSCLC patients from nine RCTs were included for analysis. The incidence of CR in NSCLC patients treated with ICIs was $1.5 \%$ (95\% CI: $0.8-3.0)$ compared to $0.7 \%$ (95\% CI: 0.4-1.2) in CT groups. The use of ICIs in advanced NSCLC significantly improved the possibility of archiving CR (RR 2.89, 95\% CI: 1.44-5.81, $P=0.003$ ) compared to CT. Subgroup analysis according to ICIs showed that the use of

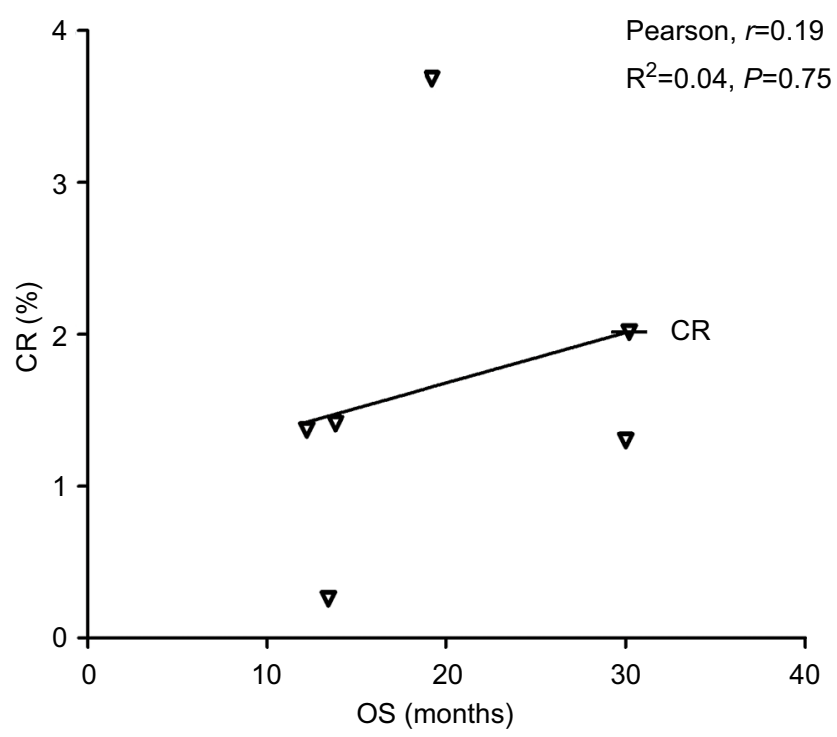

Figure 4 Correlation between median OS and CR in NSCLC patients who received ICls.

Abbreviations: $\mathrm{CR}$, complete response; $\mathrm{ICl}$, immune checkpoint inhibitor; NSCLC, non-small-cell lung cancer; OS, overall survival.

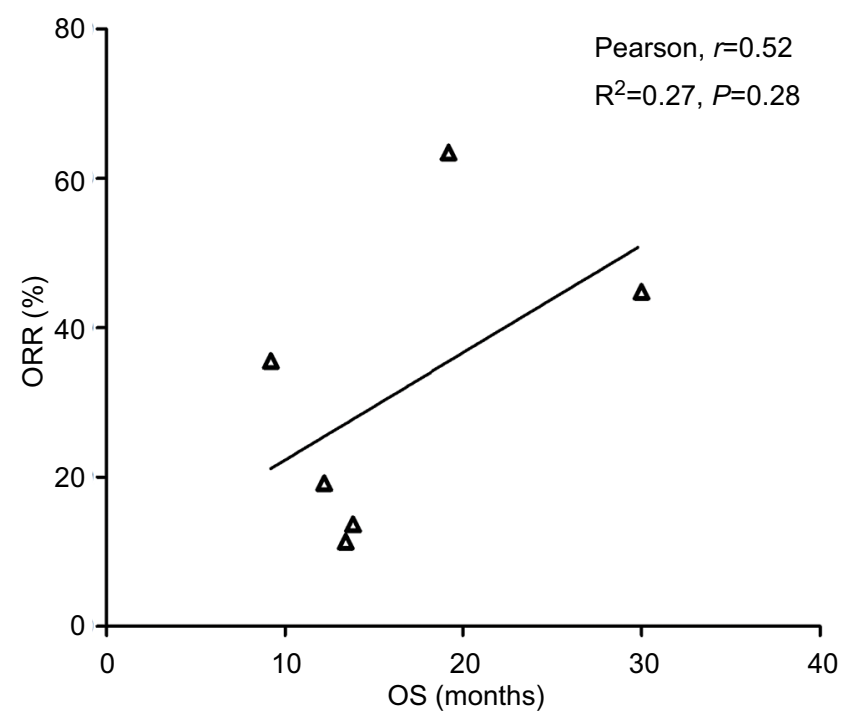

Figure 5 Correlation between median OS and ORR in NSCLC patients who received ICls.

Abbreviations: $\mathrm{ICl}$, immune checkpoint inhibitor; NSCLC, non-small-cell lung cancer; ORR, objective response rate; OS, overall survival.

atezolizumab (RR 3.26, $P=0.01$ ) and nivolumab (RR 4.83, $P=0.042$ ) in advanced NSCLC significantly improved the CR rate in comparison with $\mathrm{CT}$ alone, but not pembrolizumab and ipilimumab. We also found that the use of ICIs as first-line (RR 2.39, 95\% CI: 1.08-5.3, $P=0.032$ ) or second-line (RR 4.99, 95\% CI: $1.10-22.66, P=0.038)$ therapy significantly increased the change in obtaining a CR. In addition, correlation analysis indicates that PFS was strongly correlated with OS in NSCLC patients who received ICIs $(r=0.67$ for 
PFS, $P=0.023)$. No marked correlation was found between OS and ORR ( $r=0.48, P=0.13$ ). Based on our findings, ICIs alone or combination are recommended as initial treatment for unresectable advanced NSCLC patients in order to pave the way for potentially radical surgery of the primary and metastatic sites.

We also assessed the potential surrogate points for OS in NSCLC patients who received ICIs. Roviello et $\mathrm{al}^{36}$ previously investigated the role of the tumor RR after ICI-based therapy as a potential surrogate end point of PFS and OS in patients with solid tumors. The authors found a weak correlation between RR and OS, supporting future investigations to assess the surrogacy of RR in the patients treated with ICIs. In consistent with previous studies, no marked correlation was found between OS and CR ( $r=0.19, P=0.75)$ and OS and ORR ( $r=0.52, P=0.28)$. One potential reason for this observation is the heterogeneity of the patients' cohort as patients with different levels of PD-L1 expression were included for analysis, while current studies have demonstrated that the PD-L1 expression on formalin-fixed, paraffin-embedded tumor section by immunohistochemistry (IHC) could be a marker to identify potential responder to novel ICIs. Additionally, in the present study, we find that there is a strong correlation between OS and PFS ( $r=0.89)$, and this correlation is statistically significant $(P=0.017)$. Based on our findings, PFS appears to be a good surrogate end point for OS in NSCLC patients receiving ICIs.

The results of our meta-analysis represent the largest amount of evidence that the use of ICIs is effective at increasing the rate of CR in NSCLC patients when compared to controls. The quality of this evidence is based on the high rate of the mean Jadad score for the included studies. Nevertheless, there are several limitations that need to be mentioned. First of all, this meta-analysis only considers published literature, and lack of individual patient data prevents us from adjusting the treatment effect according to disease and patient variables. In addition, we are unable to investigate whether the ability of archiving CRs associated with ICIs is similar in NSCLC patients with or without EGFR or ALK gene mutation. Second, CR events are prospectively collected for each clinical trial, but our study is retrospective, and there are potentially important differences among the studies, which could be another source of heterogeneity. However, the pooled analysis indicates that there is no significant heterogeneity among included trials. Finally, most of included trials are open label, with an inherent risk of bias. Although the literature search is comprehensive, the possibility of relevant publication remains might not be identified.

\section{Conclusion}

Although the CR is a rare event in advanced NSCLC patients, the use of ICIs significantly increases the chance of archiving $\mathrm{CR}$ in comparison with CT. Further studies are still needed to investigate whether treatment with ICIs can be discontinued in these patients. In addition, PFS is significantly correlated with OS and could be used as a surrogate end point in patients with NSCLC who have received ICIs therapy, but not for CRs/ORR.

\section{Availability of data and material}

All data generated or analyzed during this study are included in this published article.

\section{Acknowledgments}

We would like to thank the reviewers whose comments and suggestions greatly improved this manuscript. This work was supported by the National Natural Science Foundation of China (81770028), the Natural Science Foundation of Guangdong (2016A030310087), and the Project of Shenzhen Basic Research Plan (JCYJ20160422165836057).

\section{Disclosure}

The authors report no conflicts of interest in this work.

\section{References}

1. Siegel R, Naishadham D, Jemal A. Cancer statistics, 2012. CA Cancer J Clin. 2012;62(1):10-29.

2. Bareschino MA, Schettino C, Rossi A, et al. Treatment of advanced non small cell lung cancer. J Thorac Dis. 2011;3(2):122-133.

3. Kim ES, Hirsh V, Mok T, et al. Gefitinib versus docetaxel in previously treated non-small-cell lung cancer (INTEREST): a randomised phase III trial. Lancet. 2008;372(9652):1809-1818.

4. Shepherd FA, Rodrigues Pereira J, Ciuleanu T, et al; National Cancer Institute of Canada Clinical Trials Group. Erlotinib in previously treated non-small-cell lung cancer. $N$ Engl J Med. 2005;353(2):123-132.

5. Iacono D, Chiari R, Metro G, et al. Future options for ALK-positive non-small cell lung cancer. Lung Cancer. 2015;87(3):211-219.

6. Socinski MA, Evans T, Gettinger S. Treatment of stage IV non-small cell lung cancer: Diagnosis and management of lung cancer, 3rd ed: American College of Chest Physicians evidence-based clinical practice guidelines. Chest. 2013;143(5 Suppl):e341S-e368S.

7. Asahina H, Sekine I, Horinouchi H, et al. Retrospective analysis of third-line and fourth-line chemotherapy for advanced non-small-cell lung cancer. Clin Lung Cancer. 2012;13(1):39-43.

8. Chen YM, Shih JF, Fan WC, et al. Third-line or fourth-line chemotherapy in non-small-cell lung cancer patients with relatively good performance status. J Chin Med Assoc. 2011;74(5):209-214.

9. Stinchcombe TE. The use of EGFR tyrosine kinase inhibitors in EGFR wild-type non-small-cell lung cancer. Curr Treat Options Oncol. 2016;17(4):18.

10. Lauko A, Thapa B, Venur VA, Ahluwalia MS. Management of brain metastases in the new era of checkpoint inhibition. Curr Neurol Neurosci Rep. 2018;18(10):70.

11. Lee CK, Man J, Lord S, et al. Checkpoint inhibitors in metastatic EGFR-mutated non-small cell lung cancer-a meta-analysis. $J$ Thorac Oncol. 2017;12(2):403-407. 
12. Pardoll DM. The blockade of immune checkpoints in cancer immunotherapy. Nat Rev Cancer. 2012;12(4):252-264.

13. Ribas A. Adaptive immune resistance: how cancer protects from immune attack. Cancer Discov. 2015;5(9):915-919.

14. Pu X, Wu L, Su D, Mao W, Fang B. Immunotherapy for non-small cell lung cancers: biomarkers for predicting responses and strategies to overcome resistance. BMC Cancer. 2018;18(1):1082.

15. Ribas A, Wolchok JD. Cancer immunotherapy using checkpoint blockade. Science. 2018;359(6382):1350-1355.

16. Gandhi L, Rodríguez-Abreu D, Gadgeel S, et al; KEYNOTE-189 Investigators. Pembrolizumab plus chemotherapy in metastatic nonsmall-cell lung cancer. N Engl J Med. 2018;378(22):2078-2092.

17. Vokes EE, Ready N, Felip E, et al. Nivolumab versus docetaxel in previously treated advanced non-small-cell lung cancer (CheckMate 017 and CheckMate 057): 3-year update and outcomes in patients with liver metastases. Ann Oncol. 2018;29(4):959-965.

18. Borghaei H, Paz-Ares L, Horn L, et al. Nivolumab versus docetaxel in advanced nonsquamous non-small-cell lung cancer. $N$ Engl J Med. 2015;373:1627-1639.

19. Govindan R, Szczesna A, Ahn MJ, et al. Phase III trial of ipilimumab combined with paclitaxel and carboplatin in advanced squamous nonsmall-cell lung cancer. J Clin Oncol. 2017;35(30):3449-3457.

20. Moher D, Cook DJ, Eastwood S, Olkin I, Rennie D, Stroup DF. Improving the quality of reports of meta-analyses of randomised controlled trials: the QUOROM statement. Quality of reporting of meta-analyses. Lancet. 1999;354(9193):1896-1900.

21. Cheyne WW. An address on acute abdominal symptoms: delivered before the Walthamstow division of the metropolitan counties branch of the British medical association. Br Med J. 1905;1(2320):1313-1316

22. Zintzaras E, Ioannidis JP. Heterogeneity testing in meta-analysis of genome searches. Genet Epidemiol. 2005;28(2):123-137.

23. Dersimonian R, Laird N. Meta-analysis in clinical trials. Control Clin Trials. 1986;7(3):177-188.

24. Begg CB, Mazumdar M. Operating characteristics of a rank correlation test for publication bias. Biometrics. 1994;50(4):1088-1101.

25. Egger M, Smith GD, Schneider M, Minder C. Bias in meta-analysis detected by a simple, graphical test. BMJ. 1997;315(7109):629-634.
26. Jadad AR, Moore RA, Carroll D, et al. Assessing the quality of reports of randomized clinical trials: is blinding necessary? Control Clin Trials. 1996;17(1):1-12.

27. Brahmer J, Reckamp KL, Baas P,et al. Nivolumab versus Docetaxel in Advanced Squamous-Cell Non-Small-Cell Lung Cancer. $N$ Engl J Med. 2015;373:123-135.

28. Lynch TJ, Bondarenko I, Luft A, et al. Ipilimumab in combination with paclitaxel and carboplatin as first-line treatment in stage IIIB/IV non-small-cell lung cancer: results from a randomized, double-blind, multicenter phase II study. J Clin Oncol. 2012;30(17):2046-2054.

29. Rittmeyer A, Barlesi F, Waterkamp D,et al. Atezolizumab versus docetaxel in patients with previously treated non-small-cell lung cancer (OAK): a phase 3, open-label, multicentre randomised controlled trial. Lancet. 2017;389(10066):255-265.

30. Herbst RS, Baas P, Kim DW, et al. Pembrolizumab versus docetaxel for previously treated, PD-L1-positive, advanced non-small-cell lung cancer (KEYNOTE-010): a randomised controlled trial. Lancet. 2016;387(10027):1540-1550.

31. Reck M, Rodríguez-Abreu D, Robinson AG, et al. Pembrolizumab versus Chemotherapy for PD-L1-Positive Non-Small-Cell Lung Cancer. N Engl J Med. 2016;375(19):1823-1833.

32. Hellmann MD, Ciuleanu TE, Pluzanski A, et al. Nivolumab plus ipilimumab in lung cancer with a high tumor mutational burden. $N$ Engl J Med. 2018;378(22):2093-2104.

33. Socinski MA, Jotte RM, Cappuzzo F, et al; IMpower150 Study Group. Atezolizumab for first-line treatment of metastatic nonsquamous NSCLC. N Engl J Med. 2018;378(24):2288-2301.

34. Jones CM, Brunelli A, Callister ME, Franks KN. Multimodality treatment of advanced non-small cell lung cancer: where are we with the evidence? Curr Surg Rep. 2018;6(2):5.

35. Antonia SJ, Villegas A, Daniel D, et al. Durvalumab after chemoradiotherapy in stage III non-small-cell lung cancer. $N$ Engl J Med. 2017;377(20):1919-1929.

36. Roviello G, Andre F, Venturini S, et al. Response rate as a potential surrogate for survival and efficacy in patients treated with novel immune checkpoint inhibitors: A meta-regression of randomised prospective studies. Eur J Cancer. 2017;86:257-265.
Cancer Management and Research

\section{Publish your work in this journal}

Cancer Management and Research is an international, peer-reviewed open access journal focusing on cancer research and the optimal use of preventative and integrated treatment interventions to achieve improved outcomes, enhanced survival and quality of life for the cancer patient. The manuscript management system is completely online and includes

\section{Dovepress}

a very quick and fair peer-review system, which is all easy to use. Visit http://www.dovepress.com/testimonials.php to read real quotes from published authors. 